\title{
Beneficial Effect of Efonidipine, an L- and T-Type Dual Calcium Channel Blocker, on Heart Rate and Blood Pressure in Patients With Mild-to-Moderate Essential Hypertension
}

\author{
Il-Young Oh, $\mathrm{MD}^{1}$, Myung-Ki Seo, $\mathrm{MD}^{2}$, Hae-Young Lee, $\mathrm{MD}^{2}$, Soon Gil Kim, $\mathrm{MD}^{3}$, Ki-Sik Kim, $\mathrm{MD}^{4}$, \\ Won-Ho Kim, $\mathrm{MD}^{5}$, Min Soo Hyon, $\mathrm{MD}^{6}$, Kyoo-Rok Han, $\mathrm{MD}^{7}$, Se-Joong Lim, $\mathrm{MD}^{8}$ and Cheol-Ho Kim, MD \\ ${ }^{1}$ Department of Internal Medicine, Seoul National University College of Medicine, Bundang Hospital, Seongnam, \\ ${ }^{2}$ Department of Internal Medicine, Seoul National University College of Medicine, Seoul, \\ ${ }^{3}$ Department of Internal Medicine, Hanyang University College of Medicine, Guri Hospital, Guri, \\ ${ }^{4}$ Department of Internal Medicine, Daegu Catholic University College of Medicine, Daegu, \\ ${ }^{5}$ Department of Internal Medicine, Chonbuk National University College of Medicine, Jeonju, \\ ${ }^{6}$ Department of Internal Medicine, Soonchunhyang University College of Medicine, Seoul, \\ ${ }^{7}$ Department of Internal Medicine, Hallym University College of Medicine, Seoul, \\ ${ }^{8}$ Department of Internal Medicine, Yonsei University College of Medicine, Gangnam Severance Hospital, Seoul, Korea
}

\begin{abstract}
Background and Objectives: Efonidipine hydrochloride, an L- and T-type dual calcium channel blocker, is suggested to have a heart rate (HR)-slowing action in addition to a blood pressure (BP)-lowering effect. The aim of this study was to determine the effect of efonidipine on HR and BP in patients with mild-to-moderate hypertension. Subjects and Methods: In a multi-center, prospective, open-labeled, single-armed study, we enrolled 53 patients who had mild-to-moderate hypertension \{sitting diastolic BP (SiDBP) 90-110 mmHg\}. After a 2-week washout, eligible patients were treated with efonidipine ( $40 \mathrm{mg}$ once daily for 12 weeks). The primary end point was the change in HR from baseline to week 12 . The secondary endpoint included the change in trough sitting BP and 24-hour mean BP between baseline and week 12. Laboratory and clinical adverse events were monitored at each study visit ( 4,8 , and 12 weeks). Results: Fifty-two patients were included in the intention-to-treat analysis. After 12 weeks of treatment with efonidipine, the resting HR decreased significantly from baseline to week 12 \{from $81.5 \pm 5.3$ to $71.8 \pm 9.9$ beats/minute (difference, $-9.9 \pm 9.0$ beats/minute), $\mathrm{p}<0.0001\}$. The trough BP \{sitting systolic blood pressure (SiSBP) and SiDBP $\}$ and 24-hour mean BP also decreased significantly (SiSBP: from 144.6 \pm 8.2 to 132.9 $\pm 13.5 \mathrm{mmHg}, \mathrm{p}<0.0001$; SiDBP: from $96.9 \pm 5.4$ to $88.3 \pm 8.6 \mathrm{mmHg}, \mathrm{p}<0.0001$, 24-hour mean systolic BP: from $140.4 \pm 13.5$ to $133.8 \pm 11.6 \mathrm{mmHg}$, $\mathrm{p}<0.0001$; 24 -hour mean diastolic BP: from $91.7 \pm 8.7$ to $87.5 \pm 9.5 \mathrm{mmHg}, \mathrm{p}<0.0001$ ). Conclusion: Efonidipine was effective in controlling both $\mathrm{HR}$ and $\mathrm{BP}$ in patients with mild-to-moderate hypertension. (Korean Circ J 2010;40:514-519)
\end{abstract}

KEY WORDS: Calcium channel blockers; Heart rate; Hypertension.

\section{Introduction}

Efonidipine hydrochloride, a new generation dihydropyridine (DHP) calcium channel blocker, inhibits both T-type and L-type calcium channels. ${ }^{1)}$ Typical DHP calcium antagonists, such as nifedipine and amlodipine, selectively inhibit L- type calcium channels of vascular smooth muscle cells, resulting in vasodilation. In addition to exhibiting an antihypertensive effect through vasodilation by blocking L-type calcium channels, efonidipine hydrochloride has also been suggested to regulate heart rate (HR) by inhibiting the T-type calcium channels, which are localized primarily in the sinoatrial node

Received: March 3, 2010 / Revision Received: April 2, 2010 / Accepted: April 9, 2010

Correspondence: Cheol-Ho Kim, MD, Department of Internal Medicine, Seoul National University College of Medicine, Bundang Hospital, 166 Gumi-ro, Bundang-gu, Seongnam 463-707, Korea

Tel: 82-31-787-7001, Fax: 82-31-787-4051, E-mail: cheolkim@plaza.snu.ac.kr

$@$ This is an Open Access article distributed under the terms of the Creative Commons Attribution Non-Commercial License (http://creativecommons.org/licenses/by-nc/3.0) which permits unrestricted non-commercial use, distribution, and reproduction in any medium, provided the original work is properly cited. 
and are involved in the pacemaker mechanism of the heart. ${ }^{2}$

Furthermore, efonidipine has been reported to have a negative chronotropic effect, which may be involved in controlling tachycardia. ${ }^{3)}$ Working on sinoatrial node cells by inhibiting T-type calcium channel activation, efonidipine prolongs the late phase- 4 depolarization of the sinoatrial node action potential, which suppresses an elevated HR. Previous studies have shown that efonidipine decreased the HR in contrast with other DHPs in patients with essential hypertension. ${ }^{4) 5}$

Recent epidemiologic studies have confirmed earlier studies that showed resting HR to be an independent predictor of cardiovascular and all-cause mortality, independent of gender or pre-existing cardiovascular disease. ${ }^{67)}$ Clinical trial results also suggest that reduction in HR alone is an important benefit afforded by beta-blockers in patients with angina pectoris, acute myocardial infarction, and chronic heart failure.

Regarding hypertension treatment, an approved consensus guideline has recommended antihypertensive medication with HR-lowering properties, especially in females and the elderly. ${ }^{8)}$ The aim of this study was to determine the effect of efonidipine on HR and blood pressure (BP) in patients with mild-to-moderate essential hypertension.

\section{Subjects and Methods}

\section{Patients and study design}

This study was performed with a multi-center, prospective, open-labeled, single-armed design at seven sites in the Republic of Korea. Men and women were eligible if they were 1880 years of age, had mild-to-moderate hypertension with a sitting diastolic BP (SiDBP) between 90 and $110 \mathrm{mmHg}$, a HR $<75$ beats/minute, and agreed to participate in the study. The Framingham study ${ }^{9)}$ showed that subjects with a HR $>75$ beats/ minute had higher incidence of and morbidity from cardiovascular disease than subjects with a $\mathrm{HR}<75$ beats/minute. Thus, we planned to evaluate the negative chronotropic effect of efonidipine in patients with a HR $>75$ beats/minute.

Patients were excluded if they had severe hypertension \{ sitting systolic BP (SiSBP) $\geq 180 \mathrm{mmHg}$, a difference in BP between two arms $>20 \mathrm{mmHg}$ (SiSBP) or $>10 \mathrm{mmHg}$ (SiDBP), a history of stroke or myocardial infarction in the previous 6 months, severe congestive heart failure, sick sinus syndrome or sinus bradycardia ( $<50$ beats/minute), second- or thirddegree atrioventricular block, documented hypersensitivity to DHP or efonidipine hydrochloride, hepatic disease with aspartate aminotransferase (AST) or alanine aminotransferase (ALT) $>2$ times the upper normal limit, renal disease with a serum creatinine concentration $>2.0 \mathrm{mg} / \mathrm{dL}$, uncontrolled diabetes (hemoglobin A1C >9\%), renal artery stenosis, or secondary hypertension. Women who were pregnant or breastfeeding, or who were not using an effective method of contraception, were excluded from the study. All patients gave written informed consent and the institutional review board at each center approved this study.

The study consisted of initial screening, a 2-week washout period, and a 12-week active treatment period. Complete medical history, physical examination, and laboratory tests were evaluated during the initial screening.

After the 2-week washout period of previous antihypertensive medications, patients were given $40 \mathrm{mg}$ efonidipine at 8:00 a.m. ( \pm 2 hours). If the SiSBP was $>140 \mathrm{mmHg}$ or the SiDBP was $>90 \mathrm{mmHg}$ at 4 or 8 weeks, the dosage of efonidipine was increased up to $60 \mathrm{mg}$ once daily. Clinical followup was performed at 4,8 , and 12 weeks of treatment. At each visit, the SiSBP, SiDBP, and pulse rate were measured. The resting HR was measured manually for 1 minute after the patient had rested in a sitting position for 30 minutes. The BP was measured as follows: the same time of day, before dosing, the same arm, by the same investigator at each center, and using mercury sphygmomanometers manufactured by one company (W.A. Baum Co. Inc., Copiague, NY, USA). ${ }^{10)}$ Ambulatory BP monitoring (ABPM) was performed twice at baseline and at the end of the 12-week treatment. At each visit, patients were asked about adverse events (AEs). The treatment schedule is summarized in Fig. 1.

\section{Efficacy and safety variables}

The primary efficacy variable was the mean change in resting HR from baseline to week 12 . The secondary efficacy variables included the change from baseline of the mean office trough BP (i.e., 24 hours after the last dosing) and the 24-hour mean BP assessed with ABPM after 12 weeks. The response rate was defined as the proportion of patients in which the Si-

\begin{tabular}{l}
$\begin{array}{l}\text { Visit } 1 \\
\text { (week - })\end{array}$ \\
\hline Screening/Washout
\end{tabular}

Fig. 1. Study design. SiSBP: sitting systolic blood pressure, SiDBP: sitting diastolic blood pressure. 
DBP was $<90 \mathrm{mmHg}$ or had decreased by $\geq 10 \mathrm{mmHg}$ from baseline after 12 weeks of treatment. Tolerability was assessed based on the incidence of AEs. Medical history was updated and laboratory tests were performed at each study visit. All laboratory and clinical AEs were assessed by the investigators in terms of their relationship to the study drug and intensity. Serious AEs were defined as those that were life-threatening, required hospitalization, or were associated with significant permanent disability or congenital malformation.

\section{Statistical analysis}

A sample size of 42 patients was calculated to provide $90 \%$ power to detect a 5 beat/minute difference between baseline and 12 weeks at a significance level of $\alpha=0.05$, assuming a standard deviation of 10 beats/minute. Based on a $20 \%$ predicted dropout rate, a sample size of 53 patients was required.

For the primary and secondary outcomes, intention-to-treat (ITT) and per protocol (PP) analyses were done. The ITT analysis included all patients who received $\geq 1$ dose of study medication, had a valid resting HR and BP measurement at baseline, and had $\geq 1$ valid HR and BP measurements after baseline, with missed observations imputed using the lastobservation-carried-forward method. Patients with protocol violations and those with $<75 \%$ compliance were excluded from the PP analysis. For statistical analyses of the primary and secondary endpoints, a paired t-test was used.

For a comparison of baseline characteristics, continuous variables were compared using a t-test and analysis of covariance, and discrete variables were compared using a $\chi^{2}$ test. Differences were considered statistically significant at a $\mathrm{p}<$ 0.05 . All data analyses were performed using the Statistical

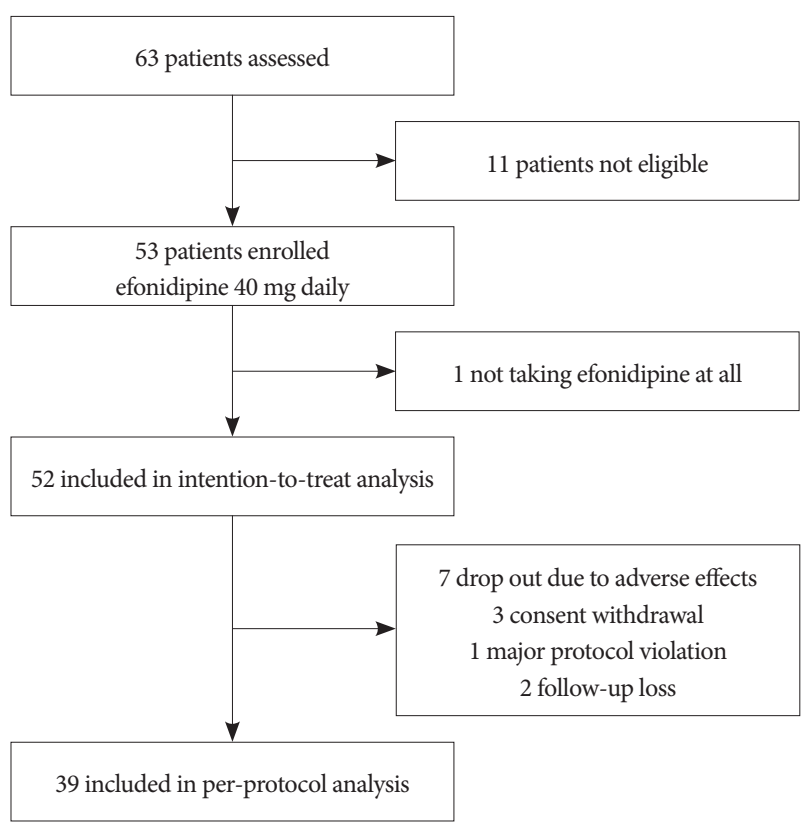

Fig. 2. Disposition of study patients.
Package for the Social Sciences (SPSS), version 13.0 (SPSS Inc., Chicago, IL, USA).

\section{Results}

\section{Patient disposition}

Sixty-three patients were screened. Eleven patients were not eligible for this study and 52 patients were enrolled in the study. After enrollment, one patient had never taken one dose of efonidipine, and was excluded from the ITT analysis. Thus, 52 patients were included in the ITT analysis. The PP analysis included 39 patients; 13 patients were excluded from the $\mathrm{PP}$ analysis due to AEs (7 patients) that included palpitations, hot flushes, nausea, headaches, dyspepsia, withdrawal of consent (2 patients), protocol violations (1 patient), and lost to follow-up (2 patients). The disposition of study patients was summarized in Fig. 2. The baseline characteristics of the enrolled patients were summarized in Table 1.

Table 1. Baseline demographic and clinical characteristics of Korean patients with mild-to-moderate essential hypertension

\begin{tabular}{|c|c|c|c|}
\hline Characteristic & $\begin{array}{c}\text { Male } \\
(\mathrm{n}=36)\end{array}$ & $\begin{array}{l}\text { Female } \\
(n=16)\end{array}$ & $\mathrm{p}$ \\
\hline Age (years) & & & 0.5552 \\
\hline Mean & $46.9 \pm 9.6$ & $48.8 \pm 13.1$ & \\
\hline Range & $31.0-65.0$ & $28.0-70.0$ & \\
\hline Height $(\mathrm{cm})$ & & & $<0.0001$ \\
\hline Mean & $170.3 \pm 4.9$ & $154.8 \pm 7.4$ & \\
\hline Range & $157.5-180.7$ & $139.5-169.7$ & \\
\hline Body weight (kg) & & & $<0.0001$ \\
\hline Mean & $73.2 \pm 9.3$ & $57.3 \pm 11.7$ & \\
\hline Range & $58.0-108.0$ & $34.0-86.4$ & \\
\hline \multicolumn{4}{|l|}{ Blood pressure (mmHg) } \\
\hline SiSBP & & & 0.8329 \\
\hline Mean & $144.8 \pm 7.8$ & $144.3 \pm 9.3$ & \\
\hline Range & $131.0-163.0$ & $131.0-160.0$ & \\
\hline SiDBP & & & 0.0518 \\
\hline Mean & $97.9 \pm 5.5$ & $94.8 \pm 4.6$ & \\
\hline Range & $90.0-109.0$ & $90.0-105.0$ & \\
\hline Smoking, no. (\%) & & & $<0.0001$ \\
\hline None & $6(16.7)$ & $14(82.4)$ & \\
\hline Previous & $10(27.8)$ & $0(0.0)$ & \\
\hline Habitual & $20(55.6)$ & $3(17.7)$ & \\
\hline Alcohol use, no. (\%) & $31(86.1)$ & $6(35.2)$ & 0.0002 \\
\hline $\begin{array}{l}\text { Previous use of } \\
\text { antihypertensives, no. (\%) }\end{array}$ & $5(13.9)$ & $2(11.8)$ & 0.8312 \\
\hline Glucose (mg/dL) & $93.7 \pm 7.7$ & $92.0 \pm 7.2$ & 0.4519 \\
\hline Creatinine (mg/dL) & $1.0 \pm 0.1$ & $0.7 \pm 0.1$ & $<0.0001$ \\
\hline Total cholesterol (mg/dL) & $195.5 \pm 27.1$ & $192.0 \pm 23.4$ & 0.9465 \\
\hline
\end{tabular}

SiSBP: sitting systolic blood pressure, SiDBP: sitting diastolic blood pressure 
Table 2. Changes in heart rate, trough SiSBP/SiDBP, and 24-hour ambulatory mean SBP/DBP from baseline to the end of treatment (week 12) with efonidipine

\begin{tabular}{|c|c|c|c|c|}
\hline & Baseline & Week 12 & Difference & $\mathrm{p}$ \\
\hline \multicolumn{5}{|c|}{ Heart rate (beats/minute) } \\
\hline Intention-to-treat & $81.5 \pm 5.3(n=52)$ & $71.8 \pm 9.9(n=48)$ & $-9.9 \pm 9.0$ & $<0.0001$ \\
\hline Per-protocol & $81.1 \pm 5.2(n=39)$ & $69.5 \pm 8.4(n=39)$ & $-11.6 \pm 8.1$ & $<0.0001$ \\
\hline \multicolumn{5}{|c|}{ Trough SiSBP (mmHg) } \\
\hline Intention-to-treat & $144.6 \pm 8.2(\mathrm{n}=52)$ & $132.9 \pm 13.5(n=48)$ & $-11.9 \pm 12.9$ & $<0.0001$ \\
\hline Per-protocol & $144.2 \pm 7.9(\mathrm{n}=39)$ & $131.3 \pm 13.1(n=39)$ & $-12.8 \pm 12.5$ & $<0.0001$ \\
\hline \multicolumn{5}{|c|}{ Trough SiDBP (mmHg) } \\
\hline Intention-to-treat & $96.9 \pm 5.4(\mathrm{n}=52)$ & $88.3 \pm 8.6(n=48)$ & $-8.8 \pm 7.7$ & $<0.0001$ \\
\hline Per-protocol & $96.7 \pm 5.3(\mathrm{n}=39)$ & $87.7 \pm 8.7(n=39)$ & $-9.0 \pm 7.8$ & $<0.0001$ \\
\hline \multicolumn{5}{|c|}{ 24-hour ambulatory mean SBP (mmHg) } \\
\hline Intention-to-treat & $140.4 \pm 13.5(n=52)$ & $133.8 \pm 11.6(n=38)$ & $-7.5 \pm 11.2$ & $<0.0001$ \\
\hline Per-protocol & $139.6 \pm 11.9(\mathrm{n}=39)$ & $133.5 \pm 11.7(\mathrm{n}=37)$ & $-6.7 \pm 10.1$ & $<0.0001$ \\
\hline \multicolumn{5}{|c|}{ 24-hour ambulatory mean DBP (mmHg) } \\
\hline Intention-to-treat & $91.7 \pm 8.7(\mathrm{n}=52)$ & $87.5 \pm 9.5(n=38)$ & $-4.3 \pm 7.2$ & $<0.0001$ \\
\hline Per-protocol & $91.5 \pm 8.8(\mathrm{n}=39)$ & $87.5 \pm 9.6(n=37)$ & $-4.5 \pm 7.2$ & $<0.0001$ \\
\hline \multicolumn{5}{|c|}{ 24-hour pulse pressure (mmHg) } \\
\hline Intention-to-treat & $48.5 \pm 11.3(\mathrm{n}=52)$ & $46.3 \pm 7.4(\mathrm{n}=38)$ & $-3.0 \pm 9.5$ & 0.0584 \\
\hline Per-protocol & $47.8 \pm 8.5(n=39)$ & $45.9 \pm 7.2(\mathrm{n}=37)$ & $-7.3 \pm 9.4$ & 0.0919 \\
\hline \multicolumn{5}{|l|}{ Non-dipper, N (\%) } \\
\hline Intention-to-treat & $30 / 52(57.7)$ & $17 / 38(44.7)$ & & \\
\hline Per-protocol & $22 / 39(56.4)$ & $16 / 37(43.2)$ & & \\
\hline
\end{tabular}

SiDBP: sitting diastolic blood pressure, SiSBP: sitting systolic blood pressure, SBP: systolic blood pressure, DBP: diastolic blood pressure

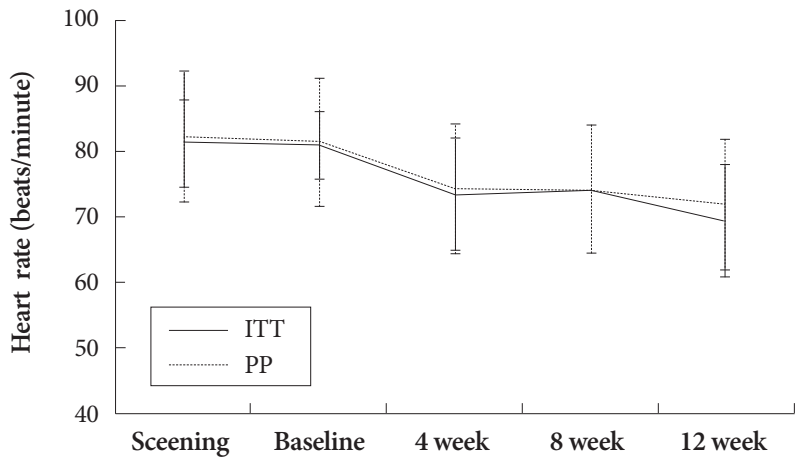

Fig. 3. Effects of efonidipine on heart rate at 4,8 , and 12 weeks. The reduction in heart rate was maintained during the active treatment period. ITT: intention-to-treat, PP: per-protocol.

\section{Change in heart rate from baseline}

The primary end point, the mean change in resting HR from baseline to week 12, was analyzed in the ITT and PP groups. Based on the ITT analysis, the resting HR decreased significantly from baseline to week 12 from $81.5 \pm 5.3$ to $71.8 \pm 9.9$ beats/minute (difference, $-9.9 \pm 9.0$ ), $\mathrm{p}<0.0001\}$ (Table 2). Based on the PP analysis, the resting HR also decreased significantly \{from $81.1 \pm 5.2$ to $69.5 \pm 8.4$ beats/minute (difference, $-11.6 \pm 8.1), \mathrm{p}<0.0001\}$. In both the ITT and PP groups, the decrease in HR was maintained during the 12-week treatment period with efonidipine (Fig. 3).

\section{Change in blood pressure from baseline}

The trough SiSBP and SiDBP decreased significantly from baseline to week 12 in both ITT and PP analyses (ITT (SiSBP), from $144.6 \pm$ to $132.9 \pm \mathrm{mmHg}, \mathrm{p}<0.0001$; ITT (SiDBP), from $96.9 \pm$ to $88.3 \pm \mathrm{mmHg}, \mathrm{p}<0.0001$; PP (SiSBP), from $144.2 \pm$ to $131.3 \pm \mathrm{mmHg}, \mathrm{p}<0.0001 ; \mathrm{PP}$ (SiDBP), from $96.7 \pm$ to $87.7 \pm$ $\mathrm{mmHg}, \mathrm{p}<0.0001\}$. The 24 -hour ambulatory mean SBP and DBP also decreased significantly in both IIT and PP analyses \{ITT (ABPM SBP), from $140.4 \pm 13.5$ to $133.8 \pm 11.6 \mathrm{mmHg}$, $\mathrm{p}<0.0001$; ITT (ABPM DBP), from $91.7 \pm 8.7$ to $87.5 \pm 9.5$ $\mathrm{mmHg}, \mathrm{p}<0.0001 ; \mathrm{PP}$ (ABPM SBP), from $139.6 \pm 11.9$ to $133.5 \pm$ $11.7 \mathrm{mmHg}, \mathrm{p}<0.0001$; $\mathrm{PP}$ (ABPM DBP), from $91.5 \pm 8.8$ to $87.5 \pm 9.6 \mathrm{mmHg}, \mathrm{p}<0.0001\}$.

\section{Response rates}

The response rates were $66.7 \%$ (32/44) for the trough SiDBP and $65.8 \%$ (25/38) for the 24-hour ambulatory mean SiDBP.

\section{Tolerability}

There was no significant difference in the vital signs between baseline and 12 weeks, except the respiratory rate. The respiratory rate was slightly increased (from $19.2 \pm 1.8$ to $19.7 \pm 1.7$ 
breaths/minute, $\mathrm{p}<0.05)$, but these changes were considered clinically insignificant. There was no significant difference in the laboratory findings between baseline and 12 weeks.

No treatment-related serious AEs requiring admission were observed. One patient was admitted to the hospital because of a ventral hernia that was considered unrelated to the study drug. Among the 52 patients, 30 AEs were observed in 17 patients (32.7\%). Eighteen AEs in 8 patients (15.4\%) were considered treatment-related. The most common $\mathrm{AE}$ was palpitations ( 6 patients), followed by facial flushing (3 patients), nausea ( 2 patients), dyspepsia ( 2 patients), headaches ( 2 patients), chest pain (1 patient), dyspnea (1 patient), and a purpuric rash (1 patient). Seven patients discontinued the study due to AEs.

\section{Discussion}

We have confirmed that efonidipine $(40-60 \mathrm{mg})$ treatment significantly reduced the HR by $11.9 \%$ in patients with mildto-moderate essential hypertension. Efonidipine also decreased the trough systolic and diastolic BP by 11.7 and 8.6 $\mathrm{mmHg}$, respectively. Efonidipine was well-tolerated.

A significant association between resting HR and allcause and cardiovascular mortality has been reported in numerous epidemiologic studies and pertains to the general population as well as patients with various cardiovascular diseases, including hypertension, acute myocardial infarction, and heart failure. ${ }^{1112)}$

In spite of excellent BP-lowering properties, the use of DHP calcium channel blockers is sometimes limited in patients with angina or myocardial infarction due to acceleration of the HR. ${ }^{13-17)}$ DHPs mainly block L-type calcium channels of blood vessels, ${ }^{18119)}$ which induces vasodilation, thus quickly reducing the $\mathrm{BP}$, but easily causes compensatory sympathetic activation, increasing the HR and cardiac oxygen demand.

However, efonidipine, which blocks both T-type and L-type calcium channels, has been reported to decrease the HR in several conditions. Morimoto et al. ${ }^{20)}$ showed that efonidipine inhibits an increase in HR and BP due to stress, presumably by blocking T-type calcium channels rather than by inhibiting the sympathetic nervous system. Tanaka et al. ${ }^{4}$ showed that efonidipine decreases the HR and plasma aldosterone more than amlodipine in patients with hypertension. Furthermore, this negative chronotropic effect has been suggested to exert an anti-arrhythmic action. Kinoshita et al. ${ }^{21)} \mathrm{dem}$ onstrated that efonidipine reduces arrhythmias and sudden death in a mouse model of dilated cardiomyopathy by repolarizing the resting membrane potential and normalizing the cardiac autonomic nervous system imbalance. These findings suggest potential therapeutic applications for efonidipine as an antihypertensive agent for patients at high risk for fatal arrhythmias and sudden death, such as patients with severe he- art failure.

Currently, the role of beta-blockers as first-line antihypertensives has been challenged. ${ }^{22)} \mathrm{A}$ meta-analysis by Lindholm et al. ${ }^{23)}$ suggested that the risk of stroke was $16 \%$ higher with beta-blockers than with other drugs and the risk of all-cause mortality was $3 \%$ higher. In fact, beta-blockers were excluded from routine initial therapy for hypertension in the 2006 British Hypertension Society guidelines. However, the HRlowering effect of beta-blockers is still required in certain hypertensive patients, such as those with high sympathetic activity.

Our findings suggest that efonidipine might be a better alternative to beta-blockers as an initial antihypertensive medication. In this study, once daily efonidipine was effective not only in the control of mild-to-moderate hypertension, but also in the reduction of HR, which is significantly associated with clinical outcome in hypertension. ${ }^{24)}$ Finally, a 12-week course of treatment with efonidipine was well-tolerated and no treatment-related serious AEs occurred.

There were several limitations to this study. First, this was an open-label, single-arm, uncontrolled study. Owing to ethical perspectives concerning non-antihypertensive therapy in patients with confirmed hypertension, we were unable to create a control group. To reduce the observer-dependent bias, we measured not only the official trough $\mathrm{BP}$, but also the ambulatory 24-hour mean BP. Second, the number of enrolled patients was too small to extrapolate to the broader population. However, our results were consistent with the previous pilot study ${ }^{5)}$ which was done in 18 patients with mild-tomoderate hypertension. This finding suggests that the beneficial effects of efonidipine on HR and BP exist.

In conclusion, efonidipine can safely decrease BP with a reduction in HR in patients with mild-to-moderate hypertension. There results suggest that efonidipine may be therapeutically useful as an initial agent for essential hypertension.

\section{Acknowledgments}

This study was supported by the Green Cross Company (Yongin, Korea).

\section{REFERENCES}

1) Tanaka H, Shigenobu K. Efonidipine hydrochloride: a dual blocker of L- and T-type ca(2+) channels. Cardiovasc Drug Rev 2002;20:81-92.

2) Hagiwara N, Irisawa H, Kameyama M. Contribution of two types of calcium currents to the pacemaker potentials of rabbit sino-atrial node cells. J Physiol 1988;395:233-53.

3) Masumiya H, Shijuku T, Tanaka H, Shigenobu K. Inhibition of myocardial L- and T-type Ca2+ currents by efonidipine: possible mechanism for its chronotropic effect. Eur J Pharmacol 1998;349:351-7.

4) Tanaka T, Tsutamoto T, Sakai H, Fujii M, Yamamoto T, Horie M. Comparison of the effects of efonidipine and amlodipine on aldosterone in patients with hypertension. Hypertens Res 2007;30:691-7.

5) Shimizu M, Ogawa K, Sasaki H, et al. Effects of efonidipine, an L- and T-Type dual calcium channel blocker, on heart rate and blood pressure in patients with mild to severe hypertension: an uncontrolled, openlabel pilot study. Current Ther Res 2003;64:707-14.

6) Diaz A, Bourassa MG, Guertin MC, Tardif JC. Long-term prognostic 
value of resting heart rate in patients with suspected or proven coronary artery disease. Eur Heart $J$ 2005;26:967-74.

7) Jouven X, Empana JP, Schwartz PJ, Desnos M, Courbon D, Ducimetiere P. Heart-rate profile during exercise as a predictor of sudden death. N Engl J Med 2005;352:1951-8.

8) Palatini P, Benetos A, Grassi G, et al. Identification and management of the hypertensive patient with elevated heart rate: statement of a European Society of Hypertension Consensus Meeting. J Hypertens 2006; 24:603-10.

9) Gillman MW, Kannel WB, Belanger A, D'Agostino RB. Influence of heart rate on mortality among persons with hypertension: the Framingham Study. Am Heart J 1993;125:1148-54.

10) Chung JW, Lee HY, Kim CH, et al. Losartan/hydrochlorothiazide fixed combination versus amlodipine monotherapy in Korean patients with mild to moderate hypertension. Korean Circ J 2009;39:151-6.

11) Fox K, Borer JS, Camm AJ, et al. Resting heart rate in cardiovascular disease. J Am Coll Cardiol 2007;50:823-30.

12) Cha DH, Cha YS, Kook JH, et al. Clinical efficacy of carvedilol in patients with moderate to severe congestive heart failure. Korean Circ J 1998;28:523-31.

13) The Israeli Sprint Study Group. Secondary prevention reinfarction Israeli nifedipine trial (SPRINT): a randomized intervention trial of nifedipine in patients with acute myocardial infarction. Eur Heart $J$ 1988;9:354-64.

14) Goldbourt U, Behar S, Reicher-Reiss H, Zion M, Mandelzweig L, Kaplinsky E. Early administration of nifedipine in suspected acute myocardial infarction. The Secondary Prevention Reinfarction Israel Nifedipine Trial 2 Study. Arch Intern Med 1993;153:345-53.

15) Furberg CD, Psaty BM, Meyer JV. Nifedipine: Dose-related increase in mortality in patients with coronary heart disease. Circulation 1995; 92:1326-31.
16) Psaty BM, Heckbert SR, Koepsell TD, et al. The risk of myocardial infarction associated with antihypertensive drug therapies. JAMA 1995;274:620-5.

17) Kim DW, Seo JD, Cho SW, et al. Antihypertensive efficacy and safety of perindopril versus nifedipine in high salt intake essential hypertensives: a double-blind parallel group study. Korean Circ J 1994; 24:156-63.

18) Furukawa T, Nukada T, Miura R, et al. Differential blocking action of dihydropyridine $\mathrm{Ca} 2+$ antagonists on a T-type Ca2+ channel (alpha1G) expressed in Xenopus oocytes. $J$ Cardiovasc Pharmacol 2005;45:241-6.

19) Furukawa T, Yamakawa T, Midera T, Sagawa T, Mori Y, Nukada T. Selectivities of dihydropyridine derivatives in blocking $\mathrm{Ca}(2+)$ channel subtypes expressed in Xenopus oocytes. J Pharmacol Exp Ther 1999;291:464-73

20) Morimoto S, Jo F, Maki K, Iwasaka T. Effects of efonidipine hydrochloride on heart rate and circulatory changes due to stress. Clin Exp Hypertens 2009;31:83-91.

21) Kinoshita H, Kuwahara K, Takano M, et al. T-type Ca2+ channel blockade prevents sudden death in mice with heart failure. Circulation 2009; 120:743-52.

22) Bloch MJ. British hypertension society recommends that beta-blockers are no longer indicated as initial treatment of hypertension: has the pendulum swung too far? J Clin Hypertens 2007;9:99-102.

23) Lindholm LH, Carlberg B, Samuelsson O. Should beta blockers remain first choice in the treatment of primary hypertension: a metaanalysis. Lancet 2005;366:1545-53.

24) Palatini P, Benetos A, Julius S. Impact of increased heart rate on clinical outcomes in hypertension: implications for antihypertensive drug therapy. Drugs 2006;66:133-44. 